\title{
A Novel Method for Predicting Facial Beauty Under Unconstrained Condition
}

\author{
Jun-ying Gan, Bin Wang, and Ying $\mathrm{Xu}^{(\bowtie)}$ \\ School of Information Engineering, Wuyi University, \\ Jiangmen 529020, Guangdong, China \\ \{junyinggan, xuyingl17\}@163.com, binw36@126.com
}

\begin{abstract}
Facial beauty prediction is a challenging task in pattern recognition and biometric recognition as its indefinite evaluation criterion, compared with the other facial analysis task such as emotion recognition and gender classification. There are many methods designed for facial beauty prediction, whereas they have some limitations. Firstly, the results are almost achieved on a relative small-scale database, thus it is difficult to model the structure information for facial beauty. Secondly, most facial beauty prediction algorithm presented previously needs burdensome landmark or expensive optimization procedure. To this end, we establish a larger database and present a novel method to predict facial beauty. The works in this paper are notably superior to previous works in the following aspects: (1) A large database is established whose distribution is more reasonable and utilized in our experiments; (2) Both female and male facial beauty are analyzed under unconstrained conditions without landmark; (3) Multi-scale apparent features are learned by our method to represent facial beauty which is more expressive and requires less computation expenditure. Experimental results demonstrate the efficacy of the presented method from the aspect of accuracy and efficiency.
\end{abstract}

Keywords: Unconstrained facial beauty prediction - Large-scale database · Multi-scale K-means $\cdot$ Spatial relationship $\cdot$ Object-part feature

\section{Introduction}

Facial beauty is an ill defined concept. It can be perceived, but can't be expressed accurately by words. What produces human perception of beauty is a long standing problem in human science and the other fields. Computer analysis of facial beauty is an emerging subject, while numerous studies have indicated that computer can learn the concept of human beauty profoundly.

Recently, researchers use geometric features [1-5], apparent features [6-8] and then predict facial beauty by machine learning methods. The former is a hotspot of research on facial beauty. Researchers extract many meaningful feature points from face images, and compute geometric distances and ratio vectors between feature points, then take them as classification features. This method is accurate and robust to lighting and background noises, but we will lose much feature information characterizing beautiful faces, such as rippling muscles, structure transition of organs, if geometric features are used simply to describe face images. Moreover, the extraction of geometric features is 
involved with a great deal of manual intervention, leading to no authoritative results. Apparent features regard the local or overall appearances of faces as research objects, which are not confined to simple quantity or proportion relationships and costly manual landmarks of facial features on the analysis of beautiful characteristics. While, if we use simple feature extraction model, many information such as spatial relationship between local structures will be ignored. Recently, deep learning catches the attention of researchers, and subsequent related research has shown that hierarchical feature possesses stronger ability of representation. Then, much recent work in this field has focused on utilizing deep models to learn better feature representation for facial beauty prediction $[6,7,9]$. These methods need vast computation expense for optimization, numerous labeled samples for fine-tuning and tricks to choose the appropriate hyper-parameters. It has been found that K-means can be used as a more effectively unsupervised feature learning method $[10,11]$. In the experimental process, we find that K-means can learn something meaningful like edge, contour, local structure like eye and nose, even face parts when the centroids learned by K-means on multi-scale image patch set are visualized. Those hierarchical object-part representations are important to facial beauty prediction as they contain spatial information between adjacent features or the other face structures, which affect facial beauty assessment heavily, and can't be represented by general appearance feature very well. On the basis of these observations, we applied K-means to extract multi-scale apparent features, called multi-scale K-means and experimental results demonstrate the efficacy of the presented approach.

Furthermore, compared with other face pattern analysis tasks, there is a central problem in this field. One is that it is difficult for people to apply an objective criterion to estimate facial beauty. The other is that it is difficult to obtain a number of extremes beauty: very attractive and very ugly. Consequently, most methods are evaluated on relatively small-scale self-established database with different rating criterion and scheme. In 2013, Mu et al. [12] established a database with 99 female images and 151 male images. In 2014, Gan et al. [6] evaluated their method on 600 female images. Yan [13] established a database with 5000 female images and 5000 male images, but its beauty distribution is not very reasonable as the extremes of facial beauty have a low proportion (about $2.4 \%$ to $4.2 \%$ ). More details about database can be consulted in [14]. In this paper, we established a large-scale database with 10000 labeled images for supervised learning and 80000 unlabeled images for unsupervised learning.

\section{Facial Beauty Database}

The database containing 5000 labeled female images, 5000 labeled male images and 80000 unlabeled human face images, some sorted examples can be found in Fig. 1. In addition, to get a good representation of beauty, we expand the original database by manually collecting extremes beauty: very attractive and extremely unattractive, and the distribution histogram is shown in Fig. 1. Each image corresponds to 20 raters, 10 males and 10 females respectively. Most rating volunteers are students and teachers aged between 20-35 years old. In the labeled database, each facial image is labeled by a discrete integer scale among 1 (extremely unattractive), 2 (unattractive), 3 (average), 4 (attractive), 5 (most attractive). 

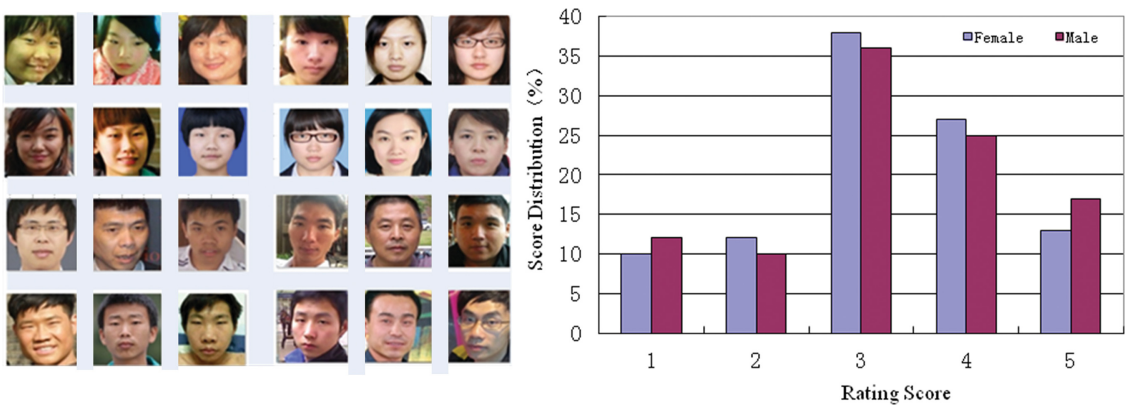

Fig. 1. Left: Some examples of our database. Right: The score distribution of database

In order to obtain scientific and reasonable rating data, the raters are randomly divided into two groups. We compute the mean ratings of each group, then calculate the Pearson correlation between two groups of mean ratings to check whether there exists a consistency between two mean ratings. This procedure repeats many times and correlation of 0.90 to 0.94 is showed between two groups of mean ratings, which indicates the consistency of ratings obtained by our assessment scheme.

\section{Presented Approach}

In this section, we will introduce the presented multi-scale model for facial beauty prediction. We present the fundamental theories briefly at first and elaborate the motivation of our method after that, then present the architecture.

\subsection{K-means Algorithm}

Given a data set $X=\left\{x^{(1)}, x^{(2)}, \cdots x^{(N)}\right\}$, where $x \in \mathrm{R}^{m}$. The classic K-means algorithm is aimed at finding $K$ centroids $\left\{\mu^{(1)}, \mu^{(2)}, \cdots, \mu^{(K)}\right\}$, so that we can partition the data set into $K$ number of clusters. The centroids can be obtained by minimizing the following formula:

$$
E=\sum_{n=1}^{N} \sum_{k=1}^{K} r_{k}^{(n)}\left\|x^{(n)}-\mu^{(k)}\right\|^{2}
$$

Where $r_{k}^{(n)}$ is an indicator describing which cluster the data point $x^{(n)}$ belongs to. It can be calculated as:

$$
r_{k}^{(n)}= \begin{cases}\text { if } & k=\arg \min _{j}\left\|x^{(n)}-\mu^{(j)}\right\|^{2} \\ 0 & \text { otherwise }\end{cases}
$$




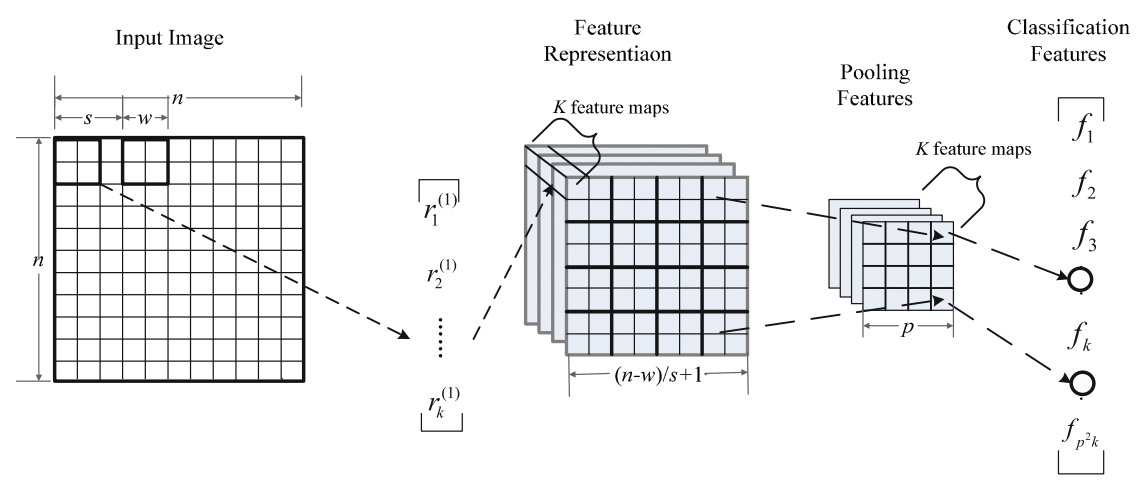

Fig. 2. Illustration of high-resolution image feature extraction by utilizing K-means. The input image consists of $n$-by- $n$ pixel, and the image patches are $w$-by- $w$.

Once we obtain the clusters and corresponding centroids, we can define a feature mapping $r: R^{m} \rightarrow R^{K}$. For any input $x^{(n)}$, it can be mapped in a vector $r^{(n)} \in R^{K}$ according to Eq. (3). This mapping method is named hard-assignment, but this method is not appropriate for sensory data with large variety in appearance [15]. There is another choice of mapping method named soft-threshold [16]:

$$
r_{k}^{(n)}=\max \left\{0, \mu(z)-z_{k}\right\}
$$

Where $z_{k}=\left\|x^{(n)}-\mu^{(k)}\right\|^{2}$ and $\mu(z)=\frac{1}{K} \sum_{k=1}^{K} z_{k}$. Compared with hard-assignment, it reserves certain sparsity and competition between features and is more suitable for object recognition.

Suppose we can learn a feature mapping $r: R^{w \times w} \rightarrow R^{K}$ by applying K-means on image patches set $\mathrm{X}$, where each image patch is $w$-by- $w$ and randomly sampled from large images. Then given a high-resolution image of $n$-by- $n$ pixels (grayscale), we can obtain a $((n-w) / s+1)$-by- $((n-w) / s+1)$-by- $K$ feature representation by utilizing feature mapping $r: R^{w \times w} \rightarrow R^{K}$ to each $w$-by-w patches of the high-resolution image, where $s$ is the step-size and $w$ is receptive field size. After that, max pooling or other pooling method are used over adjacent, disjoint spatial blocks, and regarded as classification features. The procedures are illustrated in Fig. 2.

Compared with those elaborate feature learning algorithms like sparse coding, RBM etc., K-means is more efficient since it needs less parameters to adjust and requires less computation expenditure, therefore it can be scaled up to large-scale database easily.

\subsection{Motivation and Architecture of Multi-scale K-means for Facial Beauty}

Suppose that an unlabeled image set $D_{1}$ contains 40000 images with the resolution of $120 \times 120$ randomly selected from our database. Gaussian pyramid is applied to each 
Complexity (Abstraction)

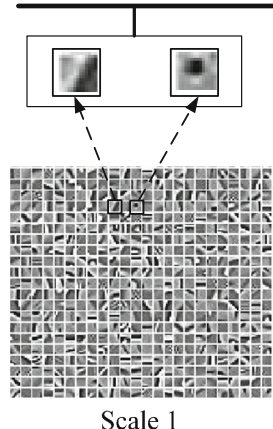

(a)

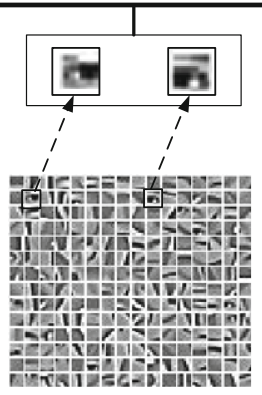

Scale 2

(b)

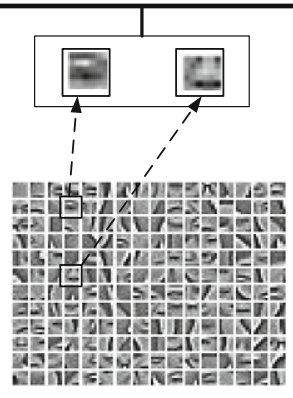

Scale 3

(c)

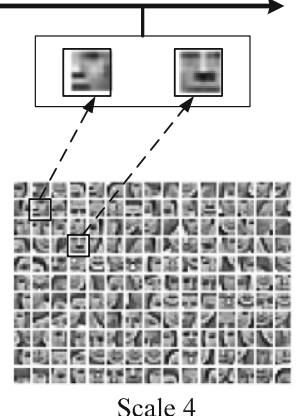

(d)

Fig. 3. Bottom: The visualization of the centroids learned on different image patch set, such as scale 1 is learned on $P_{1}$, scale 2 is learned on $P_{2}$, and so on. Top: We zoom in some centroids for better observation, and we can see that the features become more complicated from left to right.

image with four scales, namely $120 \times 120,60 \times 60,30 \times 30,15 \times 15$, so we construct the other 3 new unlabeled image sets $D_{2}, D_{3}, D_{4}$. We find that four scales are an appropriate choice on the premise of accuracy and computational efficiency. It will contain redundant feature information if overmuch scale is applied. After that, we extract 1 million image patches from each image set respectively, which can form image patch sets $P_{1}, P_{2}, P_{3}$ and $P_{4}$, just as shown in Fig. 5. Then we train K-means on those 4 image patch sets respectively, and obtained 4 groups of centroids, the visualization is shown in Fig. 3. From the visualization, we can see that K-means learned base elements like edge and corner on $P_{1}$, local structures like eye and nose on $P_{2}$, more complex structures on $P_{3}$ and face parts on $P_{4}$, and those results are similar to the high-level features presented in literature $[17,18]$.

It is shown that multi-scale K-means is more preferable for facial beauty prediction than the other feature extraction method since local feature and object-part feature can encode the spatial relationship between adjacent facial structures, which affect facial beauty assessment heavily, and can't be represented by general appearance feature well. In order to prove it, we design a simple experiment just as description in Fig. 4. In this experiment, we only adjust the space between eyes and eyebrows by image-editing tool of the original face and performance artificial rating on these two faces, and higher rating score of the original is obtained than that of the edited version. Then we compared the representational capacity between K-means and multi-scale K-means. Firstly, we performed K-means on those two images and obtained normalized code vector $V_{o}$ for original and $V_{e}$ for edited version. Secondly, $D_{\mathrm{eo}}=V_{o}-V_{e}$ was calculated and the vector $D_{e o}$ represented the degree of deviation between the corresponding code elements of two code vector. The greater the deviation, the better discrimination, as shown in Fig. 4 upper right. Then we encoded the two images by multi-scale K-means and the above step repeated, the scatter chart is shown in Fig. 4 bottom right. From the two scatter charts, we can see that multi-scale $\mathrm{K}$-means is more expressive to minor change of spatial relationship between facial structures than K-means. The more complex of 

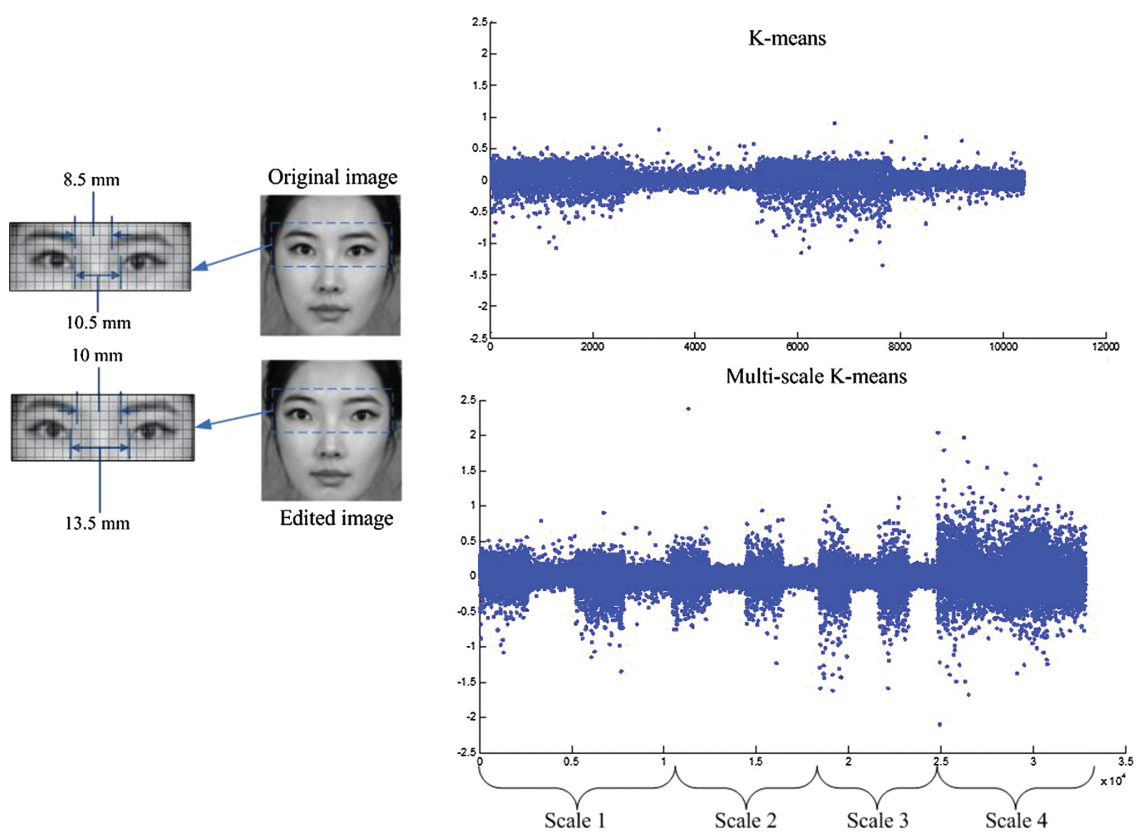

Fig. 4. Illustration of spatial information effect on facial beauty and the representation limitation of simple appearance features. The upper right is the scatter chart of the deviation of feature vectors (10400-D) of two faces encoded by K-means. The bottom right is the deviation of feature vectors (32801-D) obtained by multi-scale K-means.

the features, the more sensitive to the change, especially for scale 4 . In addition, it is also demonstrated that the complex object-part features are more selective for the specific object class $[11,17]$ and this corresponds to our experimental results.

The structure of multi-scale K-means is presented in Fig. 5. The detailed implementation is the same as described before. Notice that Kyoto images and the other nature scene images can be used to train scale 1, while we must use face images to train scale 2 to scale 4 because we want those K-means feature extractors to learn face parts instead of other uncorrelated object parts.

\section{Experimental Results and Analysis}

\subsection{Experimental Settings}

Our procedures in Matlab-2011b environments are conducted on Window 7 with Inter CPU (2 cores with each $2.1 \mathrm{GHz}$ ) and $32 \mathrm{~GB}$ RAM.

Two criteria have been applied to evaluate the performance of the presented facial beauty prediction model, and the reported results are based on the average value over 5-fold cross validation. 


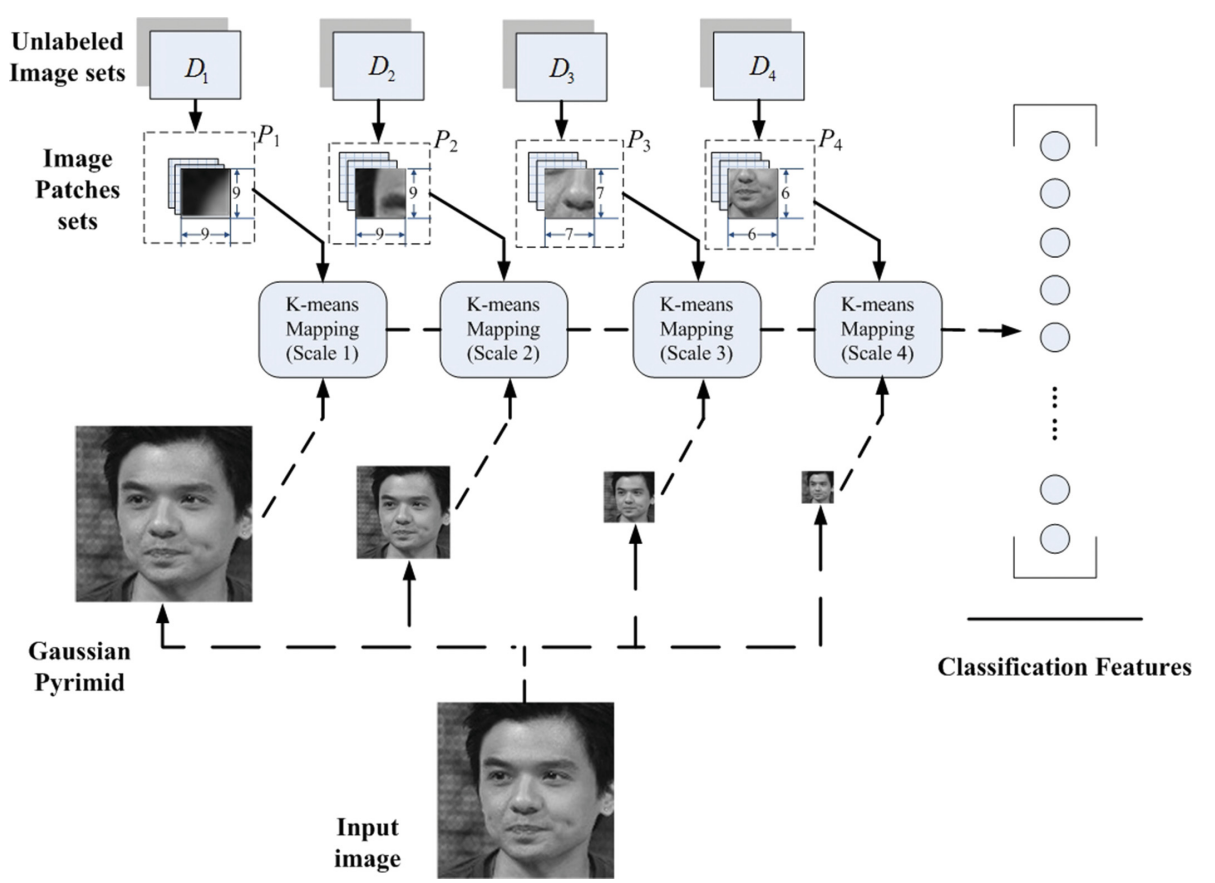

Fig. 5. Illustration of multi-scale model. The dotted lines represent feature extraction stage. The solid lines represent model training stage. The features extracted from all scales are concatenated into a single long vector and used to train a classifier.

1. Rank-1 recognition rate. It is defined as $S_{\mathrm{c}} / S_{\mathrm{t}}$, where $S_{\mathrm{c}}$ is the number of correctly classified testing samples, and $S_{\mathrm{t}}$ is the number of total testing samples.

2. F1-score. It is the harmonic mean of precision and recall and is defined as $\mathrm{F} 1=P \times R /(P+R)$, where $P$ is the precision rate, $R$ is the recall rate [19].

\subsection{Classification Performance Comparison}

We compare our method with the following feature extraction methods. The parameter settings of multi-scale K-means are shown in Table 1.

1. Raw pixel. Organize the intensity value of each pixel into a 14400-dimensional feature vector, and take it as classification features directly.

2. Eigenfaces 8. In our experiment, PCA is performed on raw pixel images, and those images are not aligned.

3. CRBM 6. We constructed CRBM to extract features for our database whose parameters setting is the same as Gan et al.

4. K-means 10. K-means had achieved state-of-the-art performance on both CIFAR-10 and NORB database, and it is the fundamental structure of our method. A K-means model with 4600 centroids is utilized here, and the other parameters are the same as scale 1 of our method. 
Table 1. The parameter setting of our method

\begin{tabular}{l|r|r|r|r}
\hline Parameter & Scale 1 & Scale 2 & Scale 3 & Scale 4 \\
\hline \# of Centroids & 2600 & 2000 & 1600 & 2000 \\
\hline Receptive field size & 9 & 9 & 7 & 6 \\
\hline Setp size & 1 & 1 & 1 & 1 \\
\hline \# of Pooling blocks & 16 & 16 & 4 & 4 \\
\hline
\end{tabular}

Table 2. Classification performance on our database

\begin{tabular}{l|l|l|l}
\hline Classifier & Feature representation & \multicolumn{2}{|l}{$\begin{array}{l}\text { Performance } \\
\text { Rank-1 recognition rate (F1-score) }\end{array}$} \\
\cline { 3 - 4 } & & Female database & Male database \\
\hline \multirow{2}{*}{ SVM } & Raw pixel & $48.31(46.54)$ & $48.65 \%(47.42 \%)$ \\
\cline { 2 - 4 } & Eigenfaces & $45.42(42.67)$ & $46.61 \%(45.51 \%)$ \\
\cline { 2 - 4 } & CRBM & $50.07(47.81)$ & $51.40 \%(49.13 \%)$ \\
\cline { 2 - 4 } & K-means & $51.14(48.33)$ & $53.24 \%(53.08 \%)$ \\
\cline { 2 - 4 } & Multi-scale K-means & $52.71(51.23)$ & $55.13 \%(54.37 \%)$ \\
\hline
\end{tabular}

From the results listed in Table 2, we make the following analysis:

1. Multi-scale K-means outperforms the other method for both female and male database. That is because our method can utilize more complicated features to encode the facial beauty information, and experimental results demonstrate the accuracy of our method.

2. The performance of eigenfaces is lower than the other methods. This is because there are various variations in our database, such as pose, lighting etc., and it affects the performance heavily.

3. K-means is a little bit better than CRBM and that is because CRBM has many hyper-parameters to be tuned, and it is difficult to obtain a set of optimal parameters without a lot of computation power.

\subsection{Classification Performance for Various Labeled Female Database}

This experiment is aimed at analyzing the relationship between the algorithm and the scale of database. We test above algorithms with 1000, 3000, 5000 labeled samples respectively.

We make the following analysis from the results displayed in Fig. 6:

1. All algorithms generally achieved performance increase by using larger database, and this is reflect the significance of data for the algorithm.

2. Larger database can compensate for the defect of model structure to some extent. As we can see that raw pixel with 5000 samples achieve the f1-measure score of $46.14 \%$, recall of $48.01 \%$ and K-means with 3000 samples achieve f1-measure score of $45.43 \%$, recall of $48.21 \%$. 

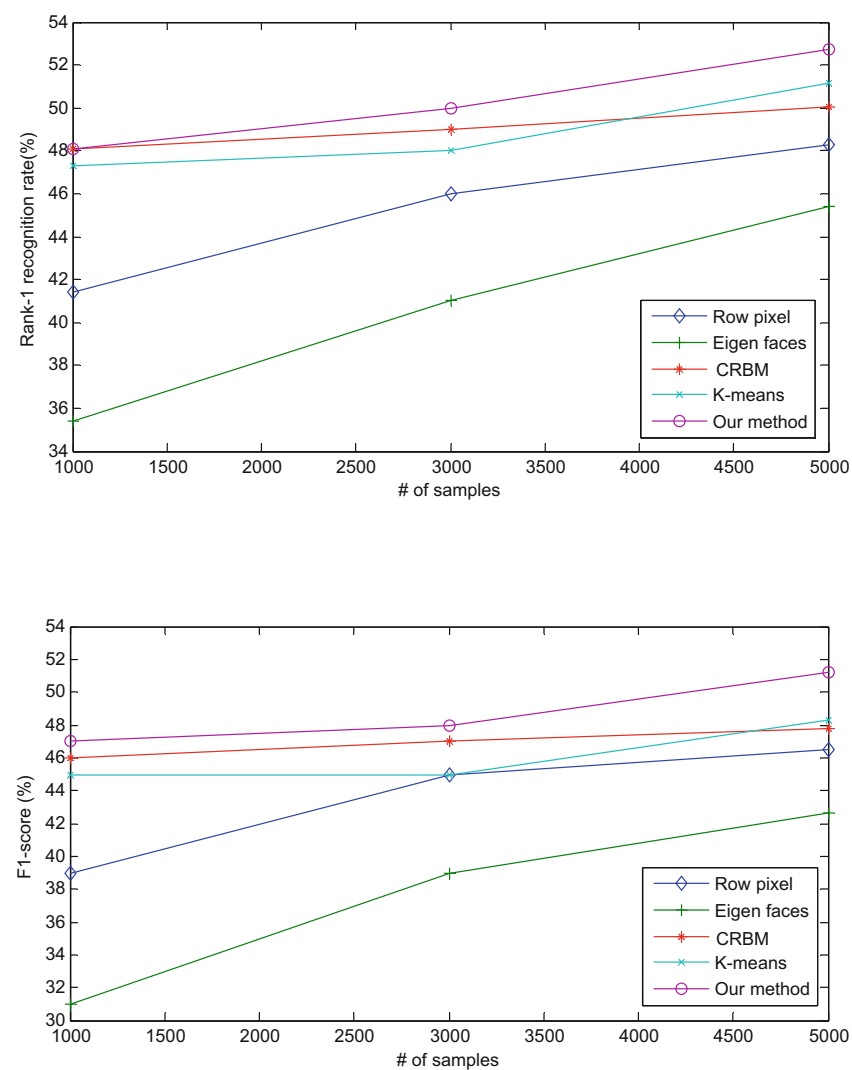

Fig. 6. Performance for various database. The top is performance based on recall rate, and the bottom is based on F1-score.

3. When labeled data is limited (e.g. total of 1000 samples) a more elaborated methods may work better. Such as compared the performance between K-means and CRBM when training them with database contains samples less than 3500 .

\subsection{Computational Time}

In this experiment, we compared cputime used by the following methods on the whole female database, in which parallel optimization is not utilized. We'll list some part of the associated settings in Table 3.

From the results listed in Table 4, we can see that cputime of CRBM for optimization stage is much larger than the other methods, while it is much less than the others for feature extraction stage, and the whole expenditure of time is about trebly the others. Our method is slightly slower than single layer K-means. This is because scale 2 , scale 3 and scale 4 of our methods are utilized to extract features for images with the 
Table 3. Parameter setting for this experiment

\begin{tabular}{l|l|l|l}
\hline Method & Epoch & \# of unlabeled samples & \# of Feature maps (centroids) \\
\hline CRBM & 20 & 10000 face images & 55 \\
\hline K-means & 50 & 1000000 patches & 4600 \\
\hline Multi-scale K-means & 50 & $\begin{array}{l}\text { Each scale } \\
1000000 \text { patches }\end{array}$ & $\begin{array}{r}\text { Scale } 1: 2600, \text { Scale 2 }: 2000, \\
\text { Scale 3 : 1600, Scale 4:2000 }\end{array}$ \\
\hline
\end{tabular}

resolution of $60 \times 60,30 \times 30,15 \times 15$ respectively and this is very fast because the data size is less compared with $120 \times 120$.

Table 4. Results of running time (in seconds)

\begin{tabular}{l|c|l|l}
\hline Method & Optimization & Feature extraction & Total \\
\hline CRBM & 44865.21 & 1690.19 & 46555.40 \\
\hline K-means & 3391.97 & 9631.92 & 13023.89 \\
\hline Multi-scale K-means & 7112.54 & 7572.64 & 14685.18 \\
\hline
\end{tabular}

\section{Conclusions}

In this paper, we contributed on two aspects. Firstly, a large-scale facial beauty database is established whose distribution is more reasonable. Secondly, a novel architecture is presented in which complex object-part feature representation is utilized maximally to encode the spatial information between features. Experimental results demonstrated the efficacy of our method on the aspect of accuracy and efficiency.

For future work, we will expand the scale of our database further and also need to improve the artificial rating scheme as we find that there are still several external influences in the experiment, such as celebrity effect, picture quality, etc. We'll analyze the performance of our method based on stacked model, and the key factors affecting facial beauty prediction from the aspect of feature representation.

Acknowledgements. This work is supported by NNSF (No. 61072127, No. 61372193, No. 61070167), NSF of Guangdong Province, P.R.C. (No. S2013010013311, No. 1015290200 1000002, No. S2011010001085, No. S2011040004211), Higher Education Outstanding Young Teachers Foundation of Guangdong Province under Grant (No. SYQ2014001) and youth foundation of Wuyi University (No. 2013zk07).

\section{References}

1. Zahang, D., Zhao, Q., Chen, F.: Quantitative analysis of human facial beauty using geometric features. Pattern Recogn. 44(4), 940-950 (2011)

2. Kagian, A., Dror, G., Leyvand, T., et al.: A machine learning predictor of facial attractiveness revealing human-like psychophysical biases. Vis. Res. 48(2), 235-243 (2008) 
3. Green, C.D.: All that glitters: a review of psychological research on the aesthetics of the golden section. Percept.-Lond. 24, 937-937 (1995)

4. Pallett, P.M., Link, S., Lee, K.: New, "golden" ratios for facial beauty. Vis. Res. 50(2), 149154 (2010)

5. Türkmen, H.Ï., Kurt, Z.: Global feature based female facial beauty decision system. In: 15th European Signal Processing Conference (EUSIPCO 2007), Poznan Poland, pp. 145-149 (2007)

6. Gan, J., Li, L., Zhai, Y., et al.: Deep self-taught learning for facial beauty prediction. Neurocomputing 144, 295-303 (2014)

7. Gray, D., Yu, K., Xu, W., Gong, Y.: Predicting facial beauty without landmarks. In: Daniilidis, K., Maragos, P., Paragios, N. (eds.) ECCV 2010, Part VI. LNCS, vol. 6316, pp. 434-447. Springer, Heidelberg (2010)

8. Eisenthal, Y., Dror, G., Ruppin, E.: Facial beauty: Beauty and the machine. Neural Comput. 18(1), 119-142 (2006)

9. Wang, J., Gong, Y., Gray, D.: Female facial beauty attribute recognition and editing. In: Fu, Y. (ed.) Human-Centered Social Media Analytics, pp. 133-148. Springer, Switzerland (2014)

10. Coates, A., Ng, A.Y., Lee, H.: An analysis of single-layer networks in unsupervised feature learning. In: International Conference on Artificial Intelligence and Statistics, pp. 215-223 (2011)

11. Weber, M., Welling, M., Perona, P.: Unsupervised learning of models for recognition. In: Vernon, D. (ed.) ECCV 2000. LNCS, vol. 1842, pp. 18-32. Springer, Heidelberg (2000)

12. $\mathrm{Mu}, \mathrm{Y} .:$ Computational facial attractiveness prediction by aesthetics-aware features. Neurocomputing 99, 59-64 (2013)

13. Yan, H.: Cost-sensitive ordinal regression for fully automatic facial beauty assessment. Neurocomputing 129, 334-342 (2014)

14. Laurentini, A., Bottino, A.: Computer analysis of face beauty: a survey. Comput. Vis. Image Underst. 125, 184-199 (2014)

15. van Gemert, J.C., Geusebroek, J.-M., Veenman, C.J., Smeulders, A.W.: Kernel codebooks for scene categorization. In: Forsyth, D., Torr, P., Zisserman, A. (eds.) ECCV 2008, Part III. LNCS, vol. 5304, pp. 696-709. Springer, Heidelberg (2008)

16. Coates, A., Ng, A.Y.: Learning feature representations with K-means. In: Montavon, G., Orr, G.B., Müller, K.-R. (eds.) Neural Networks: Tricks of the Trade, 2nd edn. LNCS, vol. 7700, pp. 561-580. Springer, Heidelberg (2012)

17. Lee, H., Grosse, R., Ranganath, R., et al.: Unsupervised learning of hierarchical representations with convolutional deep belief networks. Commun. ACM 54(10), 95-103 (2011)

18. Zeiler, M.D., Krishnan, D., Taylor, G.W., et al.: Deconvolutional networks. In: 2010 IEEE Conference on Computer Vision and Pattern Recognition (CVPR), pp. 2528-2535. IEEE (2010)

19. Powers, D.M.W.: Evaluation: from precision, recall and f-factor to ROC, informedness, markedness \& correlation. J. Mach. Learn. Technol. 2(1), 37-63 (2007) 